\title{
Keratinocyte Differentiation
}

National Cancer Institute

\section{Source}

National Cancer Institute. Keratinocyte Differentiation. NCI Thesaurus. Code C19439.

Differentiation of keratinocytes to the fully differentiated keratinocyte phenotype. 